\title{
Névralgie trigéminale essentielle
}

L'article « Névralgie trigéminale essentielle. Etude rétrospective de 35 cas 》 (Méd Buccale Chir Buccale, 2001; 7: 89-100) a suscité quelques commentaires. La plupart se trouvent exposés dans la lettre suivante adressée à la Rédaction. C'est pourquoi il semblait bon de la publier dans son intégralité. Les remarques peuvent être regroupées en deux chapitres concernant la méthologie d'une part et la discussion d'autre part.

\section{LA MÉTHODOLOGIE}

En l'absence de définition claire des critères d'inclusion dans le groupe d'étude, on ne sait si tous les signes cliniques habituellement décrits dans le tableau d'une névralgie trigéminale essentielle (NTE) étaient nécessaires à l'inclusion. Ce manque d'information paraît d'autant plus important que les critères d'exclusion pouvant renvoyer à des douleurs symptomatiques ne sont pas non plus très explicites.

L'influence de ce biais apparait déjà dans la répartition de l'échantillon : les auteurs rapportent la présence de 3 cas de névralgies atypiques avec fond douloureux permanent et celui d'une patiente pour laquelle le critère d'âge pouvait faire question. La figure 2 précise l'âge des malades lors de l'apparition des premiers symptômes : 9 malades ont moins de 50 ans - soit près de $25 \%$ de l'effectif étudié - alors que l'on sait que la survenue d'une névralgie trigéminale avant cet âge doit faire fortement suspecter son caractère symptomatique. De même, dans les résultats, les auteurs signalent des signes vasomoteurs d'accompagnement présents dans $20 \%$ des cas ce qui fait clairement poser la question de la pertinence du diagnostic (et des critères d'inclusion) puisque ce pourcentage et ces signes renvoient au syndrome SUNCT (Short Lasting Unilateral Neuralgiform headache with Conjunctival injection and Tearing) et non à une NTE $[1,2]$.

La carbamazépine a été prescrite à la dose maximale de 800 mg/jour ce qui ne correspond pas aux données récentes de la littérature puisque la dose usuelle conseillée est de $15 \mathrm{mg} / \mathrm{kg} / \mathrm{j}$ chez l'adulte. La posologie recommandée par Brasseur[3] en 1997 pouvait atteindre 2400 mg/jour dans les douleurs neuropathiques. En 2001, Vibes [4] indique que " l'expérience montre que (dans la NTE) la dose efficace se situe entre 800 et 1200 mg/jour ». C'est-à-dire précisément audessus de la dose maximale retenue par les auteurs. On peut donc se poser la question de savoir si les 23 malades sur 26 qui ont subi des infiltrations neurolytiques de glycérol n'auraient pas pu bénéficier, avant le recours à cette technique non dénuée de morbidité, d'un ajustement de leur dose quotidienne de carbamazépine selon la technique de la titration mise en œuvre avec des comprimés à 100 mg ou moins. Le recours à d'autres drogues, voire à une association, aurait pu également être discuté.

L'évaluation de la réponse au traitement n'est pas clairement explicitée dans ce travail. A aucun moment les auteurs ne précisent l'échelle d'évaluation utilisée pour apprécier la douleur initiale et le retentissement du traitement. Ils écrivent : "on peut considérer comme bons à très bons les résultats des cas... ». "On » est-ce l'opérateur ou le malade ? En terme de douleur, seul le patient est témoin de sa douleur: le recours à une échelle d'auto-évaluation est donc la règle chaque fois que l'état du malade le permet. Le taux de résultats positifs est difficilement évalué de façon fiable si l'on n'utilise pas une échelle visuelle analogique, une échelle numérique ou une échelle verbale simple. De même, alors que le «trouble psychoaffectif (a été) observé chez $65 \%$ des malades rebelles et/ou ne présentant pas de très bons résultats au traitement médical ", on ne dispose d'aucune information sur les moyens validés pour cette observation. Les questionnaires HADS (Hospital Anxiety and Depression Scale) ou l'échelle de dépression MADRS (Montgomery-Asberg Depression Rating Scale) sont, par exemple, relativement faciles à mettre en œuvre et ils ont reçu une validation internationale. Plus simplement, les auteurs omettent de préciser si le trouble « psycho-affec- médecine

buccale chirurgie buccale

VOL. $10, \mathrm{~N}^{\circ} 1$ 2004

page 7 
tif » pré-existait ou s'il était consécutif à la maladie douloureuse.

Ce défaut méthodologique aboutit à une évaluation biaisée des résultats tant par défaut que par excès : ainsi, on peut se demander si certains malades n'ont pas été sur-traités, en particulier par des injections neurolytiques, et si d'autres n'ont pas été sous-traités avec la carbamazépine, ce qui a pu conduire à la réalisation d'infiltrations neurolytiques.

\section{LA DISCUSSION}

\section{Sur le traitement médical}

Le traitement médical de la NTE n'est pas détaillé : on aurait aimé voir développer les arguments qui ont amené au traitement neurolytique lors de l'échec ou de l'épuisement de l'effet de la carbamazépine. Le clonazépam est classé dans les « thérapeutiques d'appoint » ce qui est discutable. II n'est fait aucune référence aux anticomitiaux de dernière génération (gabapentine, oxcarbazépine, lamotrigine) dont les effets antalgiques ont déjà été rapportés dans la littérature.

Dans les NTE, comme dans l'ensemble des douleurs neuropathiques centrales ou périphériques, I'hyperexcitabilité neuronale et la perturbation des échanges calciques cellulaires constituent la base du mécanisme générateur de la douleur.

L'hyperexcitabilité neuronale qui affecte toutes les voies depuis les récepteurs périphériques jusqu'au ganglion trigéminal et au cerveau, est due à plusieurs changements moléculaires : expression anormale des canaux sodium, augmentation de l'activité des sites des récepteurs du glutamate, changements dans l'inhibition de l'acide gamma-aminobutyrique, altération des flux entrants de calcium dans les cellules [5].

Ce processus est très semblable aux changements moléculaires qui affectent les cellules nerveuses au cours de l'épilepsie. C'est ce qui a inspiré l'utilisation des anticonvulsivants dans le traitement de la NTE et des douleurs neuropathiques:

- Le clonazépam, une benzodiazépine qui a des propriétés anticonvulsivantes, dispose d'une activité agoniste modulatrice sur les récepteurs post-synaptiques gabaergiques. Caccia [6] puis Court [7] ont montré son efficacité en première intention dans les douleurs de la NTE. II se révèle souvent d'un maniement plus aisé que la carbamazépine et il n'impose pas une surveillance hématologique et hépatique aussi rigoureuse. II peut être utilisé seul ou en association avec d'autres anti-épileptiques dans cette indication.

- La gabapentine qui constitue aujourd'hui une alternative de choix dans la prise en charge des douleurs neuropathiques et des douleurs de la NTE (I'AMM porte seulement sur les douleurs post-zostériennes) agit par le même mécanisme gabaergique [8]. Cette molécule a été associée avec efficacité à d'autres anticonvulsivants tels que la carbamazépine et la lamotrigine [9] chez des malades atteints de NTE au décours d'une sclérose en plaques [10]. Ces associations semblent se répandre dans la pratique clinique pour la prise en charge de NTE en état de mal ou après épuisement thérapeutique d'un anticonvulsivant isolé.

- L'oxcarbazépine est un analogue 10-kéto de la carbamazépine, mais elle possède un profil pharmacocinétique différent. En effet, alors que la carbamazépine a un métabolisme oxydatif complexe, l'oxcarbazépine est rapidement réduite en son métabolite actif, la 10,11-dihydro-10-hydroxy-carbamazépine, ce qui supprime presque complètement les risques hépatiques liés à la carbamazépine [11]. Dans les associations d'anticonvulsivants, la suppression de l'oxydation hépatique lors du remplacement de la carbamazépine par l'oxcarbazépine, semble potentialiser l'effet des autres drogues en favorisant l'élévation rapide de leur taux plasmatique, améliorant ainsi sensiblement l'effet thérapeutique global. Dès 1989, Zakrzewska[12] a publié une évaluation positive de l'effet thérapeutique de l'oxcarbazépine dans les NTE rebelles. Elle est beaucoup moins toxique et mieux tolérée, mais il semble que l'épuisement thérapeutique de l'oxcarbazépine survienne plus vite [13].

- La lamotrigine est un anticonvulsivant récent qui agit probablement en stabilisant une configuration lentement inactivée des canaux sodium et en supprimant la libération de gluta- 
mate par les neurones présynaptiques [5]. Elle a fait l'objet d'un essai en double aveugle contrôlé contre placébo chez 14 patients atteints de NTE réfractaires au tégrétol. La lamotrigine à la dose de 400 mg par jour et associée au tégrétol s'est montrée supérieure au placebo [9]. Parmi les effets secondaires de la lamotrigine, il faut néanmoins signaler la survenue de rash cutanés qui apparaissent d'autant plus facilement que la titration se fait rapidement et selon des paliers élevés [14]: c'est pourquoi la lamotrigine est exclue du traitement de crise et convient principalement à des traitements en association pour lesquels elle a été testée avec le tégretol [9], la phénytoïne [9] et la gabapentine [10].

\section{Sur les injections neurolytiques}

L'étude rétrospective de Hamdi et coll. aurait dû permettre d'évaluer les résultats thérapeutiques obtenus par l'injection du mélange éthanol-scandicaïne à l'émergence maxillaire ou mandibulaire de la branche trigéminale atteinte. Tout d'abord, on doit remarquer que le nombre de malades ayant reçu une injection (23) apparaît anormalement élevé : si les résultats du traitement médical isolé sont positifs à $88,8 \%$ (tableau 2), on comprend mal pourquoi 23 malades, soit $65 \%$ du groupe, ont reçu secondairement une injection. L'association traitement per os plus injection a permis contrôler les douleurs chez 21 patients sur 23 : comment évaluer, dans ces conditions, l'efficacité respective du traitement per os et de l'injection, en particulier en l'absence de bras placebo, dans une pathologie comme la douleur chronique, extrêmement réceptive à l'effet placebo ? Par ailleurs, le taux de récidive n'est pas commenté. Les récidives sont pourtant un écueil majeur, souvent retrouvé dans la littérature, pour les injections périphériques de glycérol ou d'alcool. Wilkinson [15] a observé un taux de récidive des névralgies de $70 \%$ à deux ans ; Fardy et coll. [16] ont constaté que la durée moyenne d'efficacité était de 13 mois après la première injection. Le traitement peut être poursuivi mais, avec les injections secondaires, on observe un épuisement progressif de l'efficacité dont le mécanisme n'est pas totalement élucidé [17].
Les injections de glycérol sont les mieux étudiées [18] : elles concernent principalement les infiltrations rétro-ganglionnaires du cavum de Meckel destinées à provoquer une véritable gangliolyse. Le glycérol est un agent neurolytique endogène qui agit comme stabilisateur de membrane en inhibant la genèse d'influx ectopiques sur le site de la lésion neuropathique. Eide et coll. [19], ont publié une étude prospective particulièrement pertinente sur des injections périphériques de glycérol au foramen infra-orbitaire et au foramen mandibulaire. Bien que cette étude porte sur des douleurs neuropathiques secondaires, et non essentielles comme dans le travail de Hamdi et coll., leurs résultats paraissent largement transposables. Les effets de l'injection périphérique de glycérol associé à un anesthésique local étaient évalués à 6 semaines après l'injection afin de distinguer l'effet de l'anesthésique de l'effet propre de la neurolyse ; les résultats sur la douleur évaluée par EVA montraient une efficacité non statistiquement significative de la neurolyse chimique. L'effet de sommation temporelle de la douleur (changements dans l'intensité de la douleur, irradiation douloureuse et altération sensitive - allodynie - postcritique) classique dans les névralgies trigéminales [20] n'était nullement modifiée par l'injection de glycérol.

En conclusion, ces auteurs [19] rappellent que, dans la littérature consacrée à la neurochirurgie, il est déconseillé d'avoir recours aux injections neurolytiques périphériques, car leur efficacité est faible et, de plus, elles peuvent entraîner des troubles sensitifs et des douleurs dysesthésiques résiduelles. L'indication des injections neurolytiques alcooliques périphériques est donc très limitée : c'est un moyen de temporiser ou de faire face aux contre-indications du traitement neurochirurgical. Même dans cette situation, il semble bien qu'on doive lui préférer le traitement médical. Enfin, la carbamazépine n'est plus le seul traitement disponible : les nouvelles molécules permettent de proposer un traitement efficace et bien toléré.

\section{Carlos MADRID}

Unité Fonctionnelle de Médecine Buccale et de Chirurgie Buccale. CHU Hôtel Dieu St Jacques. Toulouse. France médecine

buccale

chirurgie

buccale

VOL. $10, \mathrm{~N}^{\circ} 1$ 2004

page 9 


\section{RÉFÉRENCES}

1 - BOuASSIRA D. Névralgie du trijumeau et douleurs faciales atypiques in Douleurs (pp 474-492) Doin, Paris, 1997.

2 - PAREJA JA, CAMINERO AB, SJAASTAD O. SUNCT syndrome: diagnosis and treatment. CNS Drugs 2002; 16: 373-83

3 - BRASSEUR L. Traitement de la douleur (pp 104-105) Doin, Paris, 1997.

4 - VIBES J. Guide de la douleur (pp 289-296) Estem, Paris, 2001.

5 - JENSEN TS. Anticonvulsivants in neuropathic pain: rationale and clinical evidence. Eur J Pain 2002; 6 : 61-8.

6 - CACCIA MR. Clonazepam in facial neuralgia and cluster headhache: clinical and electrophysiological study. Eur Neurol 1975; 13: 560-3.

7 - COURT JE, KASE CS. Treatment of tic douloureux with a new anticonvulsivant (clonazepam). J Neurol Neurosurg Psychiatry 1976; 13: 119-22.

8 - BLOCK F. Gabapentin therapy for pain. Nervenartz 2001; 72: 69-77.

9 - ZaKrZEWSKA JM, Chaudhry Z, PATtON DW, Mullins EL. 4 Lamotrigine in refractory trigeminal neuralgia: results from a double blind placebo controlled placebo crossover study. Pain 1997; 73: 223-30.

10 - Solaro C, Messmer Ucelli M, Ucelli A, Leandri M, MANCARDI G. Low dose gabapentin combined either lamotrigine or carbamazépine can be useful for trigeminal neuralgia in multiple sclerosis. Eur Neurol 2000; 44: 45-48.

11 - Grand SM, FAULDS D, Oxcarbazepine. A review of its pharmacology and therapeutic potential in epilepsy, trigéminal neuralgia, and affective disorders. Drugs 1992; 43: 873-88.

12 - ZAKRZEWSKA JM, PASALOS PN. Oxcarbazepine: a new drug in the management of intractable trigeminal neuralgia. J Neurol Neurosurg Psychiatry 1989; 52: 472-76.
13 - ZAKRZEWSKA JM, PASALOS PN. Long term cohort study comparing medical (oxcarbazepine) and surgical management of intractable trigéminal neuralgia. Pain 2002; 95: 259-66.

14 - ZAKRZEWSKA JM HARRISON SD. Assesment and management of orofacial pain (pp 283-293) Elsevier, Amsterdam, 2002.

15 - WILKINSON HA. Trigeminal nerve peripheral branch phenol/glycerol injections for tic douloureux. J Neurosurg 1999; 90: 828-32.

16 - FARDY MJ, ZAKRZEWSKA JM, PATTON DW. Peripheral surgical techniques for the management of trigéminal neuralgia - alcohol and glycerol injections. Acta Neurochir (Wien) 1994; $129: 181-4$.

17 - AL KHATEEB TL. Morphologic effects of glycerol on the mental nerve. Oral Surg Oral Med Oral Pathol Radiol Endod 1998; 85: 248-51.

18 - STAJCIC Z. Peripheral glycerol injections in the treatment of idiopathic trigéminal neuralgia. A preliminary study. Int J Oral and Maxillofac Surg 1989; 18: 255-7

19 - Eide PK, Rabben T, SkJelbred P, Stubhaug A. The effect of the peripheral glycerol on trigéminal neuropathic pain examined by quantitative assessment of abnomal pain and sensory perception. Acta Neurochir (Wien) 1998; 140: 1271-7.

20 - EIDE PK, StubHaug A. Relief of trigeminal neuralgia after percutaneous retrogasserian glycerol rhizalysis is dependent on normalization of abnormal temporal summation of pain without general impairment of sensory perception. Neurosurg 1998; 43: 462-74. 